\title{
Using visible light links in combination with radio communication in a vehicular network
}

\author{
Fouzi Boukhalfa*, Mohamed Hadded*, Paul Muhlethaler ${ }^{\dagger}$ and Oyunchimeg Shagdar* \\ *Institute VEDECOM, 23 bis allée des Marronniers, 78000 Versailles, France \\ ${ }^{\dagger}$ EVA Team, Inria Paris, 2 Rue Simone Iff, 75012 Paris, France
}

\begin{abstract}
In VANET networks using TDMA access it is possible to approach the full capacity of the channel when the density of vehicles increases. When this occurs, some vehicles will be unable to communicate with the rest of the network. The use of Visible Light Communication (VLC) for certain vehicles can reduce the load on the radio channel and allow all vehicles to communicate on the network. The idea of our approach is to group vehicles with VLC capacity in platoons where only the lead vehicle would use radio transmission while the other vehicles in the platoon would communicate via the VLC system. We show that if the vehicles are randomly equipped with VLC transmission capabilities (with a probability p), the gain in bandwidth is significant even without rearranging the order of the vehicles. In this paper we estimate the performance gains obtained by using VLC in terms of bandwidth and probability of collision during an initial access.
\end{abstract}

Keywords - VANETs, TDMA, Active signaling, VLC, Low latency

\section{INTRODUCTION AND MOTIVATION}

The road remains a hazardous place, where many people die each year. Although improvements to the infrastructure, widespread use of the the seatbelt and new anti collision systems have all been instrumental in reducing the human toll, experience shows that despite these advances many people still die on the roads every year. Networks of vehicles, called Vehicular Adhoc NETworks (VANETs), offer great hope for reducing the number of road victims and also for improving the general conditions of road transportation. Around VANETs, several standardization initiatives have appeared in America, Europe and Japan, and inter-vehicle communications standards now exist produced by the IEEE or ETSI. Vehicular Adhoc NETworks (VANETs) have attracted considerable attention both in academic communities and industrial communities [1]. VANETs allow communications between vehicles moving along roads with the aim of establishing data exchange and collaboration between them for which different safety, commercial and entertainment applications [2] have been developed.

The existing transmission technology essentially relies on radio transmissions and a crucial aspect of communication remains the sharing of the radio channel. The solutions developed by standardization organizations in the USA and in Europe use a random access and a carrier detection technique.

Applications using VANETs often have specific and stringent QoS and security demands. Very low end-to-end latency, which can be less than $1 \mathrm{~ms}$, and a high transmission rate for huge data exchange can be required by these applications. In addition, some use cases require ultra-reliability of $99.99 \%$ [10]. Therefore, a number of Medium Access Control (MAC) [2] protocols have been proposed and developed in order to handle network access and transmission with minimum packet loss. The CSMA-based IEEE 802.11p protocol is the algorithm currently proposed to share the radio medium between users, however this protocol has been the object of much criticism, highlighting its drawbacks and limitations. In the meantime, the community has investigated access protocols based on TDMA for access in VANETs, see for instance [2] and [13]. Recently a new TDMA-based protocol, called AS-DTMAC has been proposed which combines the advantages of random access (i.e. small access delay for sporadic traffic) with the advantages of TDMA (stability of the access rights and hidden collision avoidance).

In this paper we will assume that VLC technology is available in some given vehicles. Our aim is to show how this technology can be used in place of, or in combination with, radio communication and to evaluate the gain if those vehicles use VLC instead of the radio link. The main idea we put forward in this paper is to assume that the vehicles are randomly distributed with a given probability to encompass the VLC capability. If some vehicles which follow each other have a VLC layer, they can create a platoon where the vehicles in this platoon communicate using the VLC link - the exception being the leading vehicle, which will continue to use radio communication.

The paper is organized as follows. Section II presents related work. Section III explains our assumptions and how VLC links can be created in platoons where vehicles are using a TDMA protocol. We present the protocol that enables the switch between a radio link and a VLC link. We also propose several operating modes: when the VLC links are used instead of the radio link, and when VLC links and radio transmissions are used in combination in order to improve the reliability of the network. In Section IV we study the gain that is brought by the use of the VLC communication. We will first compute the gain in the total channel load and reduction in (radio) access collisions when the nodes in the platoon switch from a radio link to a VLC link. If, on the other hand, VLC and the radio communication are used in combination we evaluate the improvement in packet delivery ratio. Section V concludes this paper. 


\section{RELATED WORK}

We find two types of related work. The first concerns communication in VANETs. The high mobility of nodes in these networks and the high variability of the vehicle density make it very difficult to ensure timely transmissions. Thus special mechanisms are necessary to meet the stringent requirements of safety applications in VANETs and we find several proposals of dedicated schemes for these applications. These proposals present special techniques to handle radio communication and can even combine the use of radio communication with VLC.

The second type of related work concerns VLC in the area of outdoor communication. Whereas indoor VLC is better established and can even reach a very high data rate, outdoor communication, where the sun can be an unavoidable noise for the communication, has been far less investigated. In this already difficult context, the mobility of nodes is an additional difficulty that must be dealt with. Below we review some studies in this field.

In [4] the authors show that the communication system is a fundamental building block to manage and maintain platoons of vehicles. To keep the system stable, strict constraints in terms of update frequency and reliability must be met. To meet these requirements, these authors develop a protocol that exploits synchronized communication slots as well as transmit power adaptation. The authors show that a combined use of a slotted scheduling mechanism and transmit power control is highly beneficial to ensure a timely transmission in the platoon, whereas dynamic approaches meant for generic information dissemination are not adequate. The content of this article confirms the idea that the communication within platoons must satisfy stringent requirements and thus must be isolated in a given communication system. to VLC transmissions.

VLC transmissions have mainly been studied for indoor transmissions without mobility and there are few studies that deal with outdoor transmissions, with or without mobility. In [5] the authors demonstrate that VLC using a single type of photodetector at the receiver can not establish a reliable link. The authors show that photodetectors with complementary properties, in terms of optical spectral response and fieldof-view, are necessary to handle the wide dynamic range of optical noise, for instance in the presence of sunlight and other unwanted light sources and with mobile users.

The advantage of VLC communications is that they operate in point-to-point and thus there is no sharing of the transmission media. This is in contrast to radio communication where a Medium Access Control (MAC) scheme is required to share the medium. The default technique used to perform this task is an adaptation of the IEEE 802.11 standard to VANETs called IEEE 802.11p [8]. The underlying technique is CSMA/CA (Carrier Sense Multiple Access with Collision Avoidance) [7] in other words the carrier must be sensed "free" before any transmission can be started. In VANETs, the continuous and fast change of network topology due to high node mobility, and the possibility of a high density of nodes make the proper functioning of IEEE 802.11p in every condition tough and they also make the design of the MAC more difficult [2]. To improve the performance of CSMA techniques, several MAC protocols for VANETs based on TDMA schemes have been proposed in the literature [11] [13] [14] [15], each of them treating a particular problem in a specific mobility scenario. However these techniques suffer from a lack of high reactivity when an urgent packet is generated in the network. The access delay is of the order of magnitude of the TDMA frames. Recently a new proposal AS-DTMAC [3] has been published which combines the advantages of a TDMA scheme but also allows emergency messages to have a very low access delay. Moreover this protocol also shows a very low access collision rate. Such collisions occur when two nodes attempt to reserve a slot in the TDMA frame.

The contribution of this paper is to show how VLC links can be used instead of, or in combination with, a radio protocol. We chose AS-DTMAC as the radio protocol used in combination with VLC. In this paper we study the benefit of having VLC communications combined with the AS-DTMAC protocol in terms of metrics such as the radio bandwidth saved, the improvement in the collision rate on the radio medium, and the improvement in packet delivery ratio.

\section{SWITCHING FROM RADIO TRANSMISSION TO VLC TRANSMISSION}

\section{A. The radio network}

Since the IEEE 802.11 p protocol suffers from a number of problems in VANETS where the density of the vehicles can vary very suddenly and where the network is very mobile, we decided to use a DTMA protocol. Our

AS-DTMAC [3] is a distributed TDMA, which is mainly based on the DTMAC protocol [11]. The main idea of the DTMAC is to divide the road into different zones $\left(x_{i}, i=\right.$ $1, \ldots, N)$ according to the communication range of the vehicles, denoted by $R$. In this way, we can impose a new concept of slot reuse, which consists in reusing the slot spatially. As described in [11], the vehicles in zone $x_{1}$ can use the same set of slots as the vehicles in $x_{4}$. The only condition to this spatial reuse is that the distance between simultaneously transmitting vehicles must be greater than $2 \times R$. Furthermore, the slot scheduling table is updated each time a packet is sent by a vehicle. The packet sent contains a special field, named Frame Information, which specifies the status of slots. Thus, it will be easy for all vehicles to select the available slots in the next frame. DTMAC acts like a slotted Aloha protocol on the 'non-busy' slots of the frame. This protocol can be applied to highway scenarios and supports different parameters (vehicles moving in opposite directions, varying speeds, varying traffic density). Thanks to GPS technology, each vehicle is able to have information about its position and the exact time. This information is useful for the functionality of DTMAC.

The new version of the protocol, called AS-DTMAC, aims to enhance the robustness of the background algorithm (DTMAC) against collisions by using the active signaling mech- 
anism. This mechanisms also allows an emergency packet to have a very low latency access. The active signaling mechanism allows the protocol to successfully preempt slots for very urgent packets. Figure 1 illustrates the inclusion of this mechanism in the slot. During the signaling part of the packet, a selection process is carried out to obtain exactly one packet to be sent in the payload part of the slot.

Signaling bursts

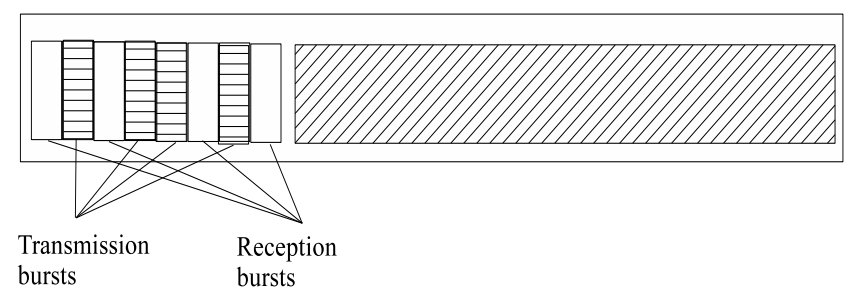

Figure 1. Slot structure of the Active Signaling mechanism

The active signaling part of the slot consists of $n$ mini-slots, each of which could be a transmission or a listening period. This succession is dictated by a randomly generated binary key. ' 1 ' means that the vehicle with a packet to send transmits during the signaling bursts. ' 0 ' means that the vehicle with a packet to send senses the channel during this mini-slot. When a vehicle selects a listening period and senses a transmission, the competition to get the slot is over. For instance, a vehicle that draws the key '01001110' will listen during the first mini-slot and if no competing transmission is sensed during this minislot, it will transmit during the next mini-slot. The following two steps in the selection process will be two listening periods. The selection process continues using the same rule until the key is completely used up.

In the description above, $n$ selection mini-slots are used in the selection process of the active signaling. In most cases we will assume that the selection key which encompasses $n$ bits is selected at random. If a vehicle has an emergency message to transmit, these keys could be handled using other techniques, in particular by creating priorities. In this case, the vehicles that require immediate access will set the first bit to ' 1 '. Thus, these vehicles will have a guaranteed priority access over the set of vehicles that are trying to get a slot using the standard scheme. These vehicles will keep the first bit set to ' 0 '.

\section{B. Using VLC communications}

We will assume that our vehicular network is composed of platoons of vehicles and thus that the communication is only required between successive vehicles. With such an assumption we can allow a car with VLC capabilities to use it instead of the radio link. We assume that the VANET is organized in platoons and that all the vehicles first use radio communication and AS-DTMAC to communicate. The idea is to build a protocol to switch vehicles using the radio link to the VLC link whenever it is possible. To perform this task we propose to use information sent in the radio packets. If we assume that the vehicles send Cooperative Awareness Messages (CAMs) we can decide to add to these messages the vehicles' VLC capability. If a vehicle has front and rear VLC modems, it indicates this in the CAM messages it periodically sends. Thus when a vehicle receives the CAMs sent by another vehicle, it can determine whether its neighboring vehicles, the one in the upstream direction and the other in the downstream direction, are equipped with VLC modems. If so, the vehicles can start to send their packets on the VLC interfaces: one to the front of the vehicle and the other to the rear. We can assume that the radio links will be kept by the vehicles until the VLC has been successfully set up by the vehicles. When the VLC links have been tested, the vehicle can stop using radio transmission and only communicate with its neighboring vehicles using the VLC link.

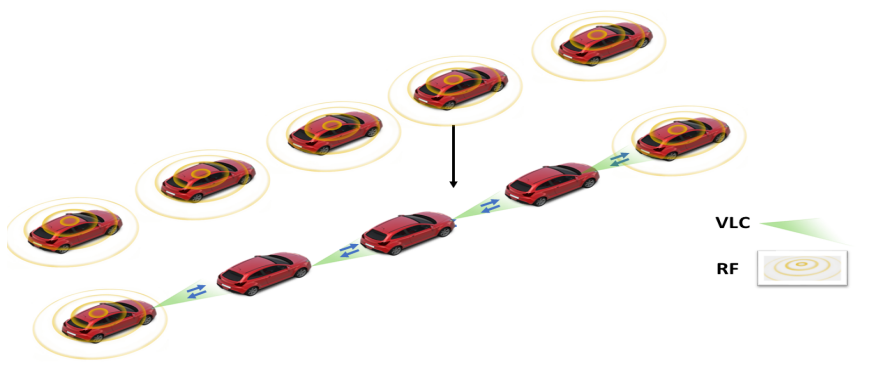

Figure 2. Switch to the VLC link

If the vehicles are not sending CAMs, then they can send special packets dedicated, for instance, to the platoon management. In these packets the vehicles will indicate their GPS position and their communication capability : radio modem, VLC modems front and/or rear. With such information, the vehicles can determine whether or not their neighboring vehicles have VLC modems and if so, start their transmission with them. Once these transmissions have been established they can give up their radio transmission. Figure 2 explains the transition from transmission using only the radio link to a combination of transmission with radio and with VLC links. The algorithm to switch from the radio to the VLC transmission is shown in Figure 3. The important point to observe is that the vehicular network must first be setup. By analyzing the CAM messages or messages for the platoon management a vehicle can determine whether two neighboring vehicles are equipped with VLC modems. If that is the case, then as soon as the VLC links of a vehicle have been established with its front and rear neighbors, the vehicle can stop using radio transmissions.

\section{Performance of the Network When the VLC IS USED}

\section{A. Gain in bandwidth}

In the following, we evaluate the gain in bandwidth if the vehicles use VLC instead of the radio link. We assume that 


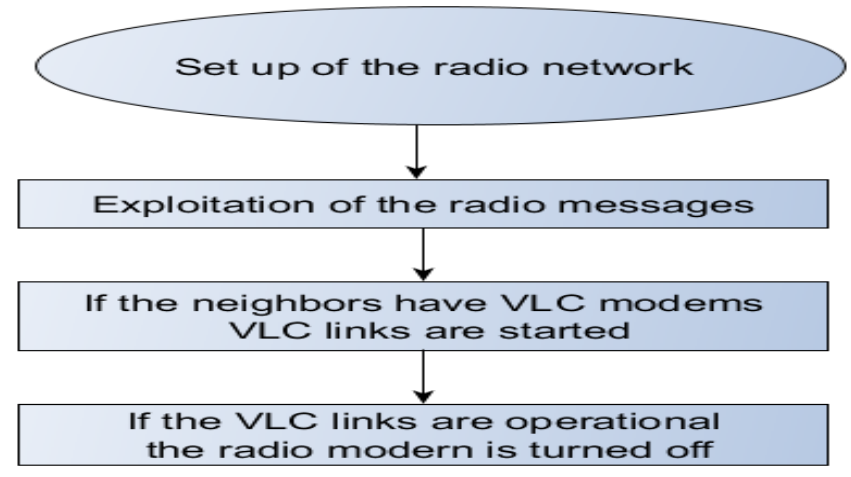

Figure 3. Algorithm to switch from the radio to the VLC link

the vehicles are equipped with VLC modems (front and rear) with a given probability $p$. We have to evaluate the number of successive vehicles with VLC capabilities in a lane of a given road. If we have $n$ successive vehicles then $n-2$ vehicles can switch from the radio modem to the VLC modem.

In the following figures we assume that if a VLC link is available, it is used instead of the radio link. In Figure 4, we present the gain in channel bandwidth when we vary the percentage of vehicles with VLC capabilities. We assume that the protocol to switch from radio communication to VLC is finished and that the VLC links have been set up without any failure. We define the gain as the percentage of vehicles that can actually use the light links instead of the radio link. We simulate this gain on a $1 \mathrm{~km}$ section of road. We observe that the gain is very notable when the percentage of vehicles equipped with VLC capabilities is greater then $50 \%$.

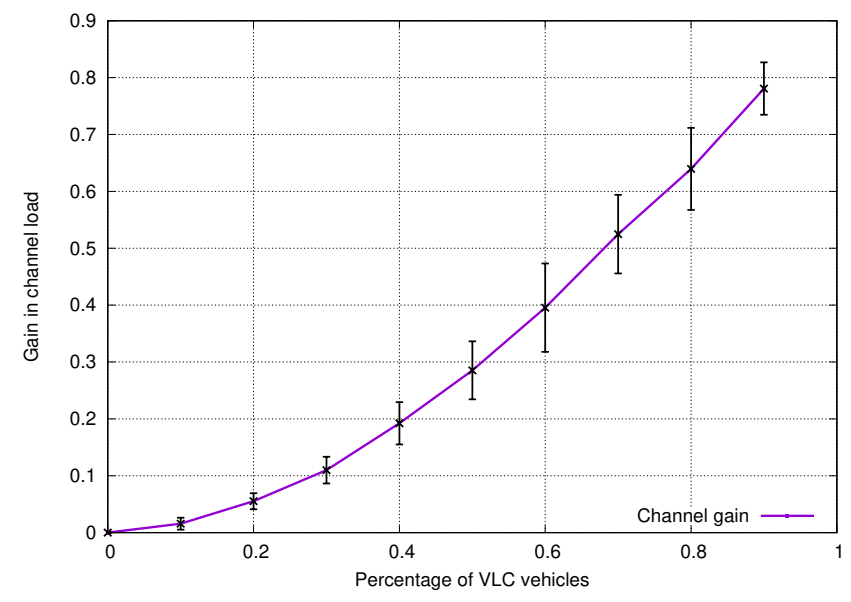

Figure 4. Percentage in channel load versus percentage of vehicles with VLC modems

In Figure 5 we present the channel load versus the vehicles inter-distance when we vary the percentage of vehicles with VLC capabilities. The vehicles are on a section of road of 1 $\mathrm{km}$ with the three sections defined in the DTMAC protocol. The length of the total frame is $0.1 \mathrm{~s}$ and the packet duration is
$1 \mathrm{~ms}$. Again, we assume that the protocol to switch from radio communication to VLC is finished and that the VLC links have been set up without any failure. We observe that the gain is very notable when the percentage of vehicles equipped with VLC capabilities is greater then $50 \%$.

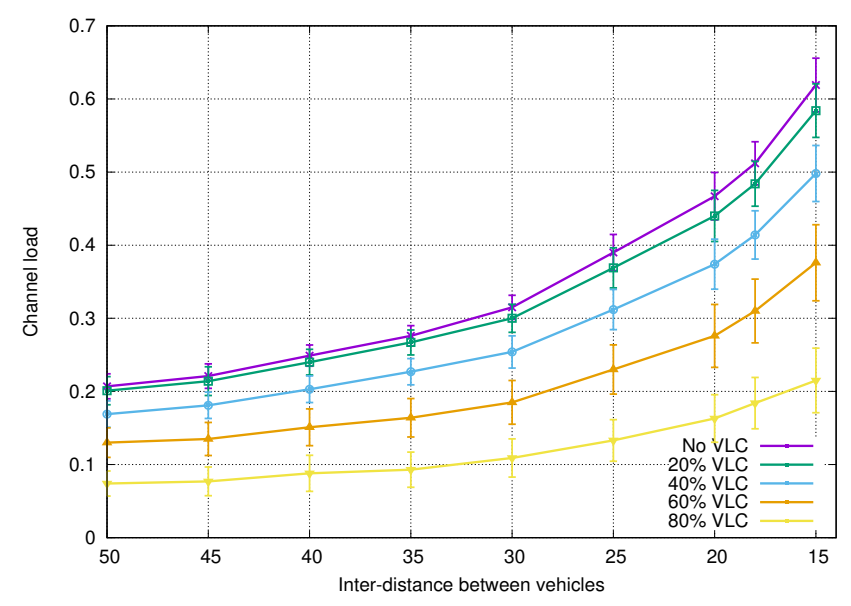

Figure 5. Channel load used versus vehicle inter-distance for different percentages of vehicles with VLC modems

\section{B. Gain in access collision probability for the radio access}

We wish to compute the gain in access collision probability for the radio access when the input load is $\lambda$. We assume that the selection process in AS-DTMAC uses $n$ signaling bursts. The access probability of AS-DTMAC for a transmission with a input load $\lambda$ is given by the following equation [6]

$P_{n}(\lambda)=\frac{1}{1-\exp (-\lambda)} \sum_{k=0}^{\infty} \frac{\lambda^{k}}{k !} \exp (-\lambda)\left(1-A_{n}^{k}(0)-\left(A_{n}^{k}\right)^{\prime}(0)\right)$.

Where the $A_{n}^{k}(x)$ are the polynomials defined by the following recursive equations

$$
A_{0}^{k}(x)=x^{k}
$$

for $i \in 0, \ldots, n-1$ :

$$
A_{i+1}^{k}(x)=A_{i}^{k}\left(\frac{x}{2}+\frac{1}{2}\right)+A_{i}^{k}\left(\frac{x}{2}\right)-A_{i}^{k}\left(\frac{1}{2}\right) .
$$

and

$$
\left(A_{n}^{k}\right)^{\prime}(0)=\frac{d A_{n}^{k}}{d x}(0) .
$$

The gain in the access collision process results from the fact that the input load $\lambda$ will become $\lambda_{1}$ with $\lambda_{1}<\lambda$. In Figure 6 we present the gain in the access collision probability when we vary the percentage of vehicles with VLC capability. We assume that $n=0$ and thus, in other words, we do not use any signaling; the radio protocol is thus only DTMAC. The load corresponds to the transmission of 1 packet of $1 \mathrm{~ms}$ every $0.1 \mathrm{~s}$. The frame encompasses 100 slots and is divided 
into three parts for three spatial sections. We assume that the inter-vehicle distance is $20 \mathrm{~m}$. We observe that the access probability decreases when the VLC links replace the radio link. Although the gain is notable, it is not extremely large.

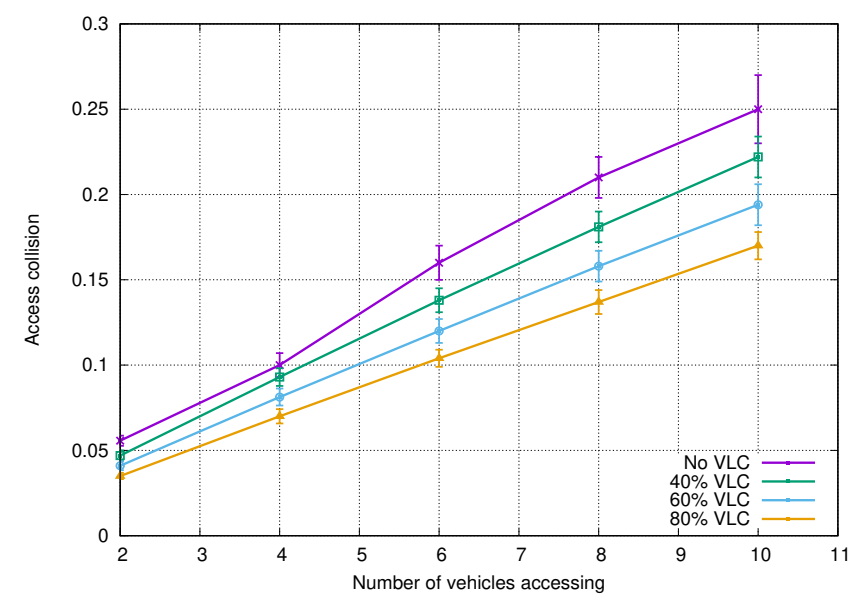

Figure 6. Channel access collision versus percentage of vehicles with VLC modems

In Figure 7 we again present the gain in the access collision probability when we vary the percentage of vehicles with VLC capability and assume that $n=10$ and the other parameters are the same as those in the previous figure. We again observe a notable decrease in the access probability when the VLC links are used instead of the radio links. However it is mostly the gain when we use signaling mechanism of AS-DTMAC that decreases the access collision probability.

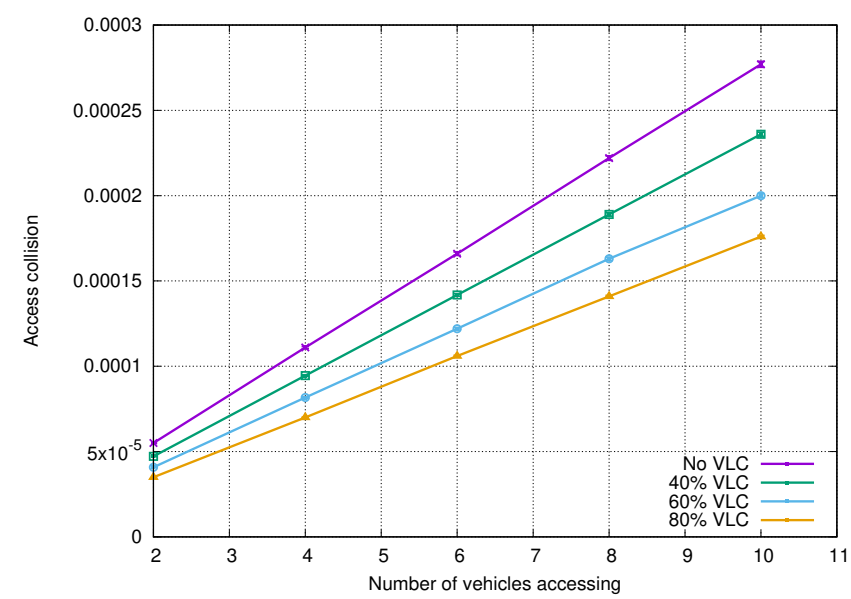

Figure 7. Channel load used versus vehicle inter-distance for different percentages of vehicles with VLC modems

\section{Gain in reliability when the radio and the VLC links are used in combination}

In this subsection we assume that the radio links and the VLC links are used simultaneously. Thus the network reliability increases since a packet loss occurs if and only if both the radio and light packets are lost. We assume that the radio link error rate is 0.0001 and that the light link error rate is 0.01 ; the errors on the two links are assumed to be independent. Thus the combination of the two links has an error rate of $10^{-6}$. In Figure 8 , we represent the mean error for a vehicular network that uses the radio and light links simultaneously versus the percentage of vehicles using the radio link. We observe that the percentage of radio links strongly determines the mean reliability of the vehicular network.

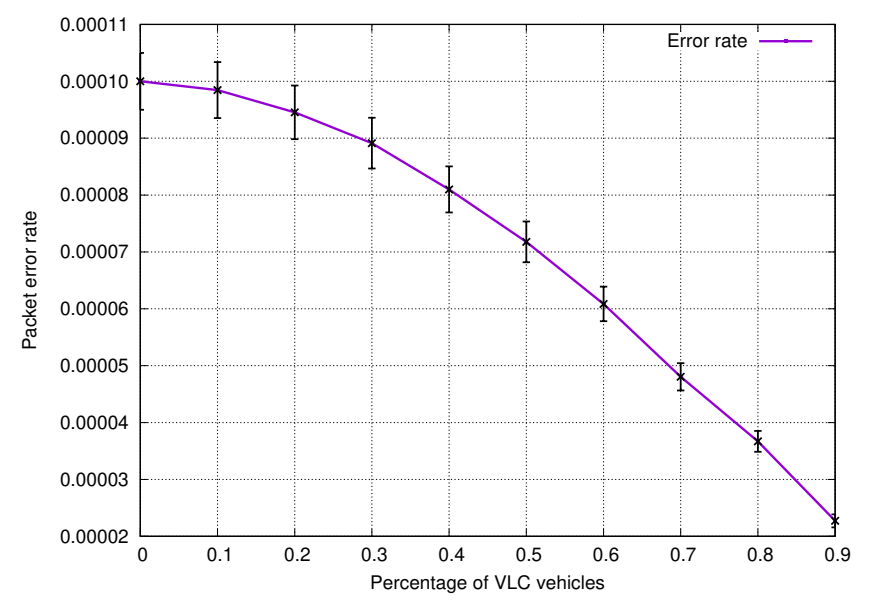

Figure 8. Packet error rate versus percentage of vehicles with VLC modems

\section{CONClusion}

We have shown how VLC can be used in combination with a vehicular radio network. We have studied how the messages sent using the radio link and which contain the vehicles' position and their VLC transmission capability, front and/or rear light modems, can be used to set up a light link between neighboring vehicles. Once the light links have been established, the radio link can be turned off and thus some radio bandwidth can be saved.

When the VLC network is used, we have studied the percentage of bandwidth used in the radio network versus the percentage of vehicles with VLC modems (front and rear). We have also studied the gain in channel load versus the percentage of vehicles with VLC modems (front and rear). We observe that the gain in bandwidth is important even if, as we have assumed, there is no re-ordering of the platoon of vehicles with VLC modems.

We have evaluated the gain for the channel collision when the VLC modems are used instead of the radio modem. We have shown that there is a notable gain when the VLC modems are used instead of the radio modem. However, an even higher gain can be obtained if we use more signaling bursts in the AS-DTMAC protocol.

The VLC modems can be used in addition to the radio links. In that case, we can increase the probability of successful transmission. A message will be lost only if both on the radio link and the VLC link are lost. We have shown that, 
in this way, we can obtain a very significant increase in the network's reliability and we have evaluated it with respect to the percentage of vehicles with VLC modems.

For future work, we need to study in greater depth how the links can be combined. Do we need a special process to control the operation of the communication modem or can we rely only on the CAM messages? We also need to better evaluate the communication loss on the two media and to determine if light links are sufficiently stable to be used instead of the radio links or whether we have to use both communication links to improve the overall reliability.

\section{REFERENCES}

[1] Fei Hu, Security and Privacy in Internet of Things (IoTs): Models, Algorithms, and Implementations, ISBN 978-1-4987-2318-3.

[2] M. Hadded, P. Muhlethaler, A. Laouiti, R. Zagrouba, and L. A. Saidane, TDMA-based MAC protocols for vehicular ad hoc networks a survey, qualitative analysis and open research issues, IEEE Communications Surveys Tutorials, vol. 17, no. 4, pp. 2461-2492, Jun. 2015.

[3] F. Boukhalfa, M. Hadded, P. Muhlethaler and O. Shagdar, An active signaling mechanism to reduce access collisions in a distributed TDMA based MAC protocol for vehicular networks" Advanced Information Networking and Applications, AINA-2019, Kunibiki Messe, Matsue, Japan March 27 to March 29, 2019

[4] M. Segata and B. Bloessl and S. Joerer and C. Sommer and M. Gerla and R. Cigno and F. Dressler, Towards inter-vehicle communication strategies for platooning support 7th International Workshop on Communication Technologies for Vehicles (Nets4Cars-Fall) 2014,

[5] Qing. Wang and Domenico. Giustiniano and Marco. Zuniga. In Light and In Darkness, In Motion and In Stillness: A Reliable and Adaptive Receiver for the Internet of Lights. IEEE Journal on Selected Areas in Communications. PP. 1-1. 10.1109/JSAC.2017.2774422.
[6] Fouzi Boukhalfa, Mohamed Hadded, Paul Muhlethaler, Oyunchimeg Shagdar. Performance Evaluation of an Active Signaling based Time-Slot Scheduling Scheme for connected vehicles Annals of Telecommunications - annales des télécommunications, Springer, 2020

[7] F. Ye, R. Yim, J. Zhang, and S. Roy, Congestion control to achieve optimal broadcast efciency in vanets, in IEEE International Conference on Communications (ICC), Cape Town, South Africa, May 2010, pp. 1-5.

[8] 802.11p-2010, IEEE standard for information technology - Telecommunications and information exchange between systems - local and metropolitan area networks - specific requirements part 11 : Wireless LAN medium access control (MAC) and physical layer (PHY) and physical layer (PHY) specifications amendment 6 : Wireless access in vehicular environments Std., 2010.

[9] R. Uzcategui and G. Acosta-Marum, Wave: A tutorial, IEEE Communications Magazine, vol. 47, no. 5, pp. 126-133, May 2009.

[10] G. Naik,B. Choudhury and J. M Park, IEEE 802.11 bd \& $5 G$ NR V2X: Evolution of Radio Access Technologies for V2X Communications, IEEE Access journal, vol. 7, pp. 70169-70184, May. 2019.

[11] M. Hadded, A. Laouiti, P. Muhlethaler, and L. A. Saidane, An infrastructure-free slot assignment algorithm for reliable broadcast of periodic messages in vehicular ad hoc networks, in Vehicular Technology Conference VTC-Fall, Montreal, Canada, Sep. 2016.

[12] F. Borgonovo, A. Capone, M. Cesana, and L. Fratta, Rr-aloha, a reliable r-aloha broadcast channel for ad-hoc intervehicle communication networks, in IEEE IFIP Annual Mediterranean Ad Hoc Networking Workshop (Med-Hoc-Net), Baia Chia, Italy, 2002.

[13] W. Zhuang, H. A. Omar, and L. Lio, Vemac: A novel multichannel mac protocol for vehicular ad hoc networks, in IEEE Conference on Computer Communications Workshops (INFOCOM WKSHPS), Shanghai, China, Aug. 2011, pp. 413-418.

[14] Y. Weidong, L. Pan, L. Yan, and Z. Hongsong, Adaptive TDMA slot assignment protocol for vehicular ad-hoc networks, Journal of China Universities of Posts and Telecommunications, vol. 20, no. 1, pp. 11-18, Feb. 2013.

[15] W. Ke, Y. Weidong, L. Pan, and Z. Hongsong, A decentralized adaptive TDMA scheduling strategy for VANETs, in IEEE Wireless Communications and Networking Conference Workshops (WCNCW), Shanghai, China, Apr. 2013, pp. 216-221. 